\title{
An Intersection Theorem for Systems of Sets
}

\author{
A. V. Kostochka* \\ Institute of Mathematics, Siberian Branch, Russian Academy of Sciences, \\ Universitetski, pr. 4, 630090 Novosibirsk, Russia
}

\section{ABSTRACT}

Erdös and Rado defined a $\Delta$-system, as a family in which every two members have the same intersection. Here we obtain a new upper bound on the maximum cardinality $\varphi(n, q)$ of an $n$-uniform family not containing any $\Delta$-system of cardinality $q$. Namely, we prove that, for any $\alpha>1$ and $q$, there exists $C=C(\alpha, q)$ such that, for any $n$,

$$
\varphi(n, q) \leq C n !\left(\frac{(\log \log \log n)^{2}}{\alpha \log \log n}\right)^{n} .
$$

(C) 1996 John Wiley \& Sons, Inc.

\section{INTRODUCTION}

Erdós and Rado [3] introduced the notion of a $\Delta$-system. They called a family $\mathscr{H}$ of finite sets a $\Delta$-system if every two members of $\mathscr{H}$ have the same intersection.

Let $\varphi(n, q)$ (respectively, $\varphi(n, q, p)$ ) denote the maximum cardinality of an $n$-uniform family not containing any $\Delta$-system of cardinality $q$ (respectively, $n$-uniform family not containing any $\Delta$-system of cardinality $q$ such that there are no $p$ pairwise disjoint sets).

Erdős and Rado [3] proved that

* This work was partially supported by the Grant 93-01-01486 of the Russian Foundation of Fundamental Research and Grant RPY300 from the International Science Foundation and Russian Government.

Random Structures and Algorithms, Vol. 9, Nos. 1 and 2 (1996)

(C) 1996 John Wiley \& Sons, Inc. CCC 1042-9832/96/010213-09 


$$
(q-1)^{n} \leq \varphi(n, q) \leq(q-1)^{n} n !\left\{1-\sum_{t=1}^{n-1} \frac{t}{(t+1) !(q-1)^{t}}\right\}
$$

and conjectured that

$$
\varphi(n, 3)<c^{n} \quad \text { for some absolute constant } c .
$$

Abbott, Hanson, and Sauer [1] improved the upper bound in (1) down to

$$
\varphi(n, q) \leq(n+1) !\left\{\frac{q-1+\left(q^{2}+6 q-7\right)^{0.5}}{4}\right\}^{n} .
$$

We are interested in asymptotic bounds on $\varphi(n, q)$ for fixed $q$. The best published upper bound of this kind is due to Spencer [5]: For fixed $q, \epsilon>0$, there exists $C=C(q, \epsilon)$ such that

$$
\varphi(n, q)<C(1+\epsilon)^{n} n !,
$$

and

$$
\varphi(n, 3)<e^{c n^{3 / 4}} n ! .
$$

Füredi and Kahn (see [2]) proved that

$$
\varphi(n, 3)<e^{c \sqrt{n}} n ! .
$$

In [4], it was proved that, for any integer $\alpha>1$, there exists $C=C(\alpha)$ such that, for any $n$,

$$
\varphi(n, 3) \leq C n ! \alpha^{-n}
$$

It appeared that the bound can be extended from $q=3$ to any fixed $q$ (using ideas of Spencer [5]). The aim of the present paper is to prove:

Theorem 1. Let $n \geq 1$ and $q \geq p \geq 2$ be integer and $\alpha(n, q)=$ $\max \left\{20, \frac{\log \log n}{20 q \log \log \log n}\right\}$. Then there exists $C(q)$ such that for all $n$,

$$
\varphi(n, q, p) \leq C(q) n ![\log \log n\rfloor^{4 p \alpha \alpha(n, q)} \alpha(n, q)^{-n} .
$$

Maybe more visual is the following immediate consequence of Theorem 1.

Corollary 2. For each integers $q>2$ and $\alpha>1$, there exists $D(q, \alpha)$ such that, for all $n$,

$$
\varphi(n, q) \leq D(q, \alpha) n !\left(\frac{(\log \log \log n)^{2}}{\alpha \log \log n}\right)^{n} .
$$

To derive Corollary 2 from Theorem 1 , consider arbitrary integers $q>2$ and $\alpha>1$. Let $n_{0}=\min \{\mid \log \log \log n>400 q \alpha\}$. Then $\alpha(n, q)=\frac{\log \log n}{20 q \log \log \log n}$, and, by Theorem 1 ,

$$
\begin{aligned}
\varphi(n, q) & =\varphi(n, q, q) \leq C(q) n !(\log \log n)^{\frac{\log \log n}{\log \log \log n}}\left(\frac{20 q \log \log \log n}{\log \log n}\right)^{n} \\
& <C(q) n !(\log n)^{1 / 5}\left(\frac{(\log \log \log n)^{2}}{2 \alpha \log \log n}\right)^{n} \leq C(q) n !\left(\frac{(\log \log \log n)^{2}}{\alpha \log \log n}\right)^{n}
\end{aligned}
$$


Choosing $D(n, \alpha)=\max \left\{C(q), \varphi\left(n_{0}, q\right)\right\}$, we get Corollary 2 .

The proof of Theorem 1 almost completely repeats that in [4]. In particular, in the course of proofs some inequalities are true when $n$ is large in comparison with $q$. We choose $C(q)$ so that the statement of the theorem holds for smaller $n$.

All the logarithms throughout the paper are taken to the base $e$.

\section{PRELIMINARY LEMMAS}

Call a family $\mathscr{F}$ of sets a $(q, n, k, p)$-family if it is an $n$-uniform family not containing any $\Delta$-system of cardinality $q$ such that the cardinality of the intersection of each two members of $\mathscr{F}$ is at most $n-k$ and there are no $p$ pairwise disjoint members of $\mathscr{F}$.

Lemma 1. For any $(q, n, k, q)$-family $\mathscr{F}$,

$$
|\mathscr{F}| \leq(q-1)^{n-k+1} \frac{n !}{k !} .
$$

Proof. We use induction on $n-k$. Any $(q, k, k, q)$-family has at most $q-1$ members. Hence the lemma is true for $n-k=0$.

Let the lemma be valid for $n-k \leq m-1$ and $\mathscr{F}$ be a $(q, m+k, k, q)$-family. Choose $q-1$ members $A_{1}, \ldots, A_{q-1}$ of $\mathscr{F}$ with maximum cardinality of their union and let $Z=\bigcup_{i=1}^{q-1} A_{i}$. Then each $A \in \mathscr{F}$ has a nonempty intersection with $Z$.

For any $x \in Z$, let $\mathscr{F}(x)=\{A \in \mathscr{F} \mid x \in A\}, \tilde{\mathscr{F}}(x)=\{A \backslash\{x\} \mid A \in \mathscr{F}(x)\}$. Then, for all $x \in Z, \tilde{\mathscr{F}}(x)$ is a $(q, m+k-1, k, q)$-family. Thus,

$$
|\tilde{F}| \leq \sum_{x \in Z}|\tilde{\mathscr{F}}(x)| \leq|Z|(q-1)^{m} \frac{(m-1+k) !}{k !} \leq(q-1)^{m+1} \frac{(m+k) !}{k !} .
$$

From now on, we suppose that for each $m \leq n-1$ and $1<p^{\prime}<p$,

$$
\begin{aligned}
& \varphi(m, q) \leq C(q) m !\lfloor\log \log m\rfloor^{4 q \alpha(m, q)} \alpha(m, q)^{-m}, \\
& \varphi\left(n, q, p^{\prime}\right) \leq C(q) n !\lfloor\log \log n\rfloor^{4 p^{\prime} \alpha(n, q)} \alpha(n, q)^{-n} .
\end{aligned}
$$

The following observation from [5] will be used throughout the paper. Let $B_{1}, \ldots, B_{t}$ be pairwise disjoint finite sets and $\mathscr{F}$ be a $(q, n, 1, q)$-family such that $\left|A \cap B_{i}\right| \geq b_{i}$ for each $A \in \mathscr{F}$. Then

$$
|\mathscr{F}| \leq\left(\begin{array}{c}
\left|B_{1}\right| \\
b_{1}
\end{array}\right) \ldots \ldots\left(\begin{array}{c}
\left|B_{t}\right| \\
b_{t}
\end{array}\right) \varphi\left(n-b_{1}-\cdots-b_{t}, q\right) .
$$

Lemma 2. Let $0<r \leq k \leq n / 2$ and for any members $A_{1}, \ldots, A_{r}$ of $a$ $(q, n, 1, q)$-family $\mathscr{F}$,

$$
\left|A_{1} \cup \cdots \cup A_{r}\right| \leq r n-k r^{2} / 2
$$

Then

$$
|\mathscr{F}| \leq C(q) \frac{n !}{k !}
$$


Proof. For $r=1$ the lemma is valid since (5) is impossible as $r=1$. Suppose that the lemma is true for $r \leq s-1$ and $|\mathscr{F}|>C(q) n ! / k$ !. By the induction hypothesis, there exist $A_{1}, \ldots, A_{s-1} \in \mathscr{F}$ such that for the set $B=A_{1} \cup \cdots \cup A_{s-1}$, we have $|B|>(s-1) n-k(s-1)^{2} / 2$. If the lemma does not hold for $\mathscr{F}$, then, for all $A \in \mathscr{F}$,

$$
|A \cap B|>b+\left((s-1) n-k(s-1)^{2} / 2\right)-\left(s n-k s^{2} / 2\right)=k(s-1 / 2),
$$

and there is an $i, 1 \leq i \leq s-1$ such that $\left|A \cap A_{i}\right|>k$. Thus, by (4),

$$
\begin{aligned}
|\mathscr{F}| \leq & (s-1)\left(\begin{array}{c}
n \\
k+1
\end{array}\right) \varphi(n-k-1, q) \\
\leq & (s-1)\left(\begin{array}{c}
n \\
k+1
\end{array}\right)\lfloor\log \log (n-k-1)\rfloor^{4 q \alpha(n-k-1, q)} \\
& \times C(q)(n-k-1) ! \alpha(n-k-1, q)^{-n+k+1} \\
\leq & \left.C(q) n ! \alpha(n-k-1, q)^{-n+k+1}\lfloor\log \log (n-k-1)\rfloor^{4 q \alpha(n-k-1, q}\right) \frac{s-1}{(k+1) !} .
\end{aligned}
$$

Note that for sufficiently large $n-k-1$, we have $\lfloor\log \log (n-k-$ $1)\rfloor^{4 q \alpha(n-k-1, q)} \leq(\log (n-k-1))^{1 / 5}$ and hence $|\mathscr{F}|<C(q) n ! / k !$.

Lemma 3. Let $\xi \geq 2,1 \leq t<s \leq n$ and $\mathscr{F}$ be a $(q, s, 1, q)$-family with $|\mathscr{F}| \geq$ $C s ! \xi^{-s}$. Then there exist $\mathscr{F}^{\prime} \subset \mathscr{F}$ and $X$ such that

(1) $|X|=s-t$

(2) for all $A \in \mathscr{F}^{\prime}, A \supset X$;

(3) $\left|\mathscr{F}^{\prime}\right| \geq C t ! \beta^{-t}$, where $\beta=(2 q \xi)^{s / t}$.

Proof. Case 1. For any $A \in \mathscr{F},|\{B \in \mathscr{F}|| B \cap A \mid \geq s-t\}| \leq C t ! 2^{s} \beta^{-t}-1$. Let $\mathscr{H}$ be a maximal $(q, s, t+1, q)$-subfamily of $\mathscr{F}$. By the choice of $\mathscr{H}$, for each $B \in \mathscr{F} \backslash \mathscr{H}$, there is an $A \in \mathscr{H}$ with $|B \cap A| \geq s-t$. Thus, $|\mathscr{F} \backslash \mathscr{H}| \leq|\mathscr{H}|\left(C t ! 2^{s} \beta^{-t}-\right.$ 1), and

$$
|\mathscr{H}| \geq \frac{|\mathscr{F}|}{C t ! 2^{s} \beta^{-t}}=\frac{s !(2 q \xi)^{s}}{t !(2 \xi)^{s}}=q^{s} \frac{s !}{t !} .
$$

But the existence of such a big $(q, s, t+1, q)$-family contradicts Lemma 1 .

Case 2. There exists $A \in \mathscr{F}$ such that $|\{B \in \mathscr{F}|| B \cap A \mid \geq s-t\}| \geq\left\lfloor C t ! 2^{s} \beta^{-t}\right\rfloor$. If $\left\lfloor C t ! 2^{s} \beta^{-t}\right\rfloor \leq 1$ then the statement is trivial. Otherwise, for some $X \subset A$ with $|X|=s-t$,

$$
|\{B \in \mathscr{F} \mid B \cap A \supset X\}| \geq\left\lfloor C t ! 2^{s} \beta^{-t}\right\rfloor\left(\begin{array}{c}
s \\
s-t
\end{array}\right)^{-1}>C t ! \beta^{-t} .
$$

This is the family we need. 


\section{MAIN CONSTRUCTION}

Let $\mathscr{F}$ be a $(q, n, 1, p)$-family with $|\mathscr{F}|=\varphi(n, q, p)$. Assume that

$$
|\mathscr{F}|>C(q) n !\lfloor\log \log n\rfloor^{4 p \alpha(n, q)} \alpha(n, q)^{-n} .
$$

The idea is to find a (not too large) family of collections of pairwise disjoint (and considerably small) sets such that most members of $\mathscr{F}$ intersect each set from at least one of these collections and then apply (4). We put

$$
\begin{gathered}
\alpha=\lfloor\alpha(n, q)\rfloor, \quad y=\lfloor n / 3 \alpha\rfloor, \quad m=3 \alpha-1, \\
k=\left\lceil\frac{n}{\log n}(\log \log n)^{3}\right\rceil, \quad r=\lfloor\log \log n\rfloor .
\end{gathered}
$$

Lemma 4. For all $s=0,1, \ldots, m$ and for $i_{0}=1$ and any $i_{1}, \ldots, i_{s} \in\{1, \ldots, r\}$ there are subfamilies $\mathscr{F}\left(1, i_{1}, \ldots, i_{s}\right)$ of the family $\mathscr{F}$ and sets $X\left(i_{1}, \ldots, i_{s}\right)$ and $Z\left(1, i_{1}, \ldots, i_{s-1}\right)$ such that, for $s \neq 0$ and for any $i_{1}, \ldots, i_{s}, i_{s}^{\prime} \in\{1, \ldots, r\}$,

(i) $\mathscr{F}\left(1, i_{1}, \ldots, i_{s}\right) \subset \mathscr{F}\left(1, i_{1}, \ldots, i_{s-1}\right)$,

(ii) for all $A \in \mathscr{F}\left(1, i_{1}, \ldots, i_{s}\right), A \supset X\left(i_{1}\right) \cup X\left(i_{1}, i_{2}\right) \cup \cdots \cup X\left(i_{1}, i_{2}, \ldots, i_{s}\right)$,

(iii) the sets $X\left(i_{1}\right), X\left(i_{1}, i_{2}\right), \ldots, X\left(i_{1}, i_{2}, \ldots, i_{s}\right)$ are pairwise disjoint,

(iv) $\left|X\left(i_{1}, i_{2}, \ldots, i_{s}\right)\right|=y$,

(v) $\left|Z\left(1, i_{1}, i_{2}, \ldots, i_{s-1}\right)\right| \leq k r(r+1) / 2$,

(vi) $X\left(i_{1}, i_{2}, \ldots, i_{s-1}, i_{s}\right) \cap X\left(i_{1}, i_{2}, \ldots, i_{s-1}, i_{s}^{\prime}\right) \subset Z\left(1, i_{1}, i_{2}, \ldots, i_{s-1}\right)$,

(viii) $\left|\mathscr{F}\left(1, i_{1}, \ldots, i_{s}\right)\right| \geq C(n-s y) ! \xi_{s}^{s y-n}$, where

$$
\xi_{s}=\left((2 \alpha)^{\frac{n}{n-s y}}(4 q)^{\frac{n+(n-y)+\cdots+(n-(s-1) y)}{n-s y}}\right)=\left((2 \alpha)^{n}(4 q)^{n s-s(s-1) y / 2}\right)^{\frac{1}{n-s y}} .
$$

Proof. We use induction on $s$. Put $\mathscr{F}(1):=\mathscr{F}, \xi_{0}:=2 \alpha$.

Steps $(0 \leq s<m)$. We have at hand $\mathscr{F}\left(1, i_{1}, \ldots, i_{s}\right)$ for any $i_{1}, \ldots, i_{s} \in$ $\{1, \ldots, r\}$ and if $s>0$ we also have sets $X\left(i_{1}, \ldots, i_{s}\right)$ and $Z\left(1, i_{1}, \ldots, i_{s-1}\right)$ as needed. Consider

$$
\begin{aligned}
\tilde{\mathscr{F}} & =\tilde{\mathscr{F}}\left(1, i_{1}, \ldots, i_{s}\right) \\
& =\left\{A \backslash\left(X\left(i_{1}\right) \cup X\left(i_{1}, i_{2}\right) \cup \cdots \cup X\left(i_{1}, i_{2}, \ldots, i_{s}\right)\right) \mid A \in \mathscr{F}\left(1, i_{1}, \ldots, i_{s}\right)\right\} .
\end{aligned}
$$

According to the statements of the lemma, $\tilde{\mathscr{F}}$ is a $(q, n-s y, 1, q)$-family. Note that $n-m y \geq n-(3 \alpha-1) n /(3 \alpha)=n /(3 \alpha)$ and hence for each $0 \leq s \leq m$,

$$
(n s-s(s-1) y / 2) /(n-s y) \leq n s /(n-m y) \leq \frac{m m}{n /(3 \alpha))}<(3 \alpha)^{2} .
$$

Therefore, for each $0 \leq s \leq m$.

$$
\xi_{s} \leq(2 \alpha)^{3 \alpha}(4 q)^{9 \alpha^{2}} \leq(8 q)^{9 \alpha^{2}}
$$

and due to Statement (vii) of the lemma, we can use Lemma 3. This Lemma 3 provides that there exists $X_{1}$ of cardinality $y$ and $\mathscr{H}_{1} \subset \tilde{\mathscr{F}}$ with $\left|\mathscr{H}_{1}\right| \geqslant C(n-(s+$ 1) $y) ! \beta^{(s+1) y-n}$ (where $\beta=\left(2 q \xi_{s}\right)^{\frac{n-s y}{n-(s+1) y}}$ ) such that each $A \in \mathscr{H}_{1}$ contains $X_{1}$. We put $Z_{1}:=\emptyset$.

Suppose that $(q, n-s y, 1, q)$-families $\mathscr{H}_{1}, \ldots, \mathscr{H}_{l}$ and sets $X_{1}, \ldots, X_{l}$, $Z_{1}, \ldots, Z_{l}$ are constructed and that, for each $1 \leq j \leq l, 1 \leq j^{\prime} \leq l, j \neq j^{\prime}$, 
- $\left|X_{j}\right|=y$,

- $\left|Z_{l}\right| \leq k l(l-1) / 2$,

- $X_{j} \cap X_{j} \subset Z_{l}$,

- for all $A \in \mathscr{H}_{j}, X_{j} \subset A$,

- $\left|H_{j}\right| \geq C(n-(s+1) y) ! \xi_{s+1}^{(s+1) y-n}$.

If $l<r$, then we construct $\mathscr{H}_{l+1}, X_{l+1}$, and $Z_{l+1}$ as follows. Note that, for each $A \in \tilde{\mathscr{\mathscr { F }}}$, we have

$$
|A|=n-s y \geq 2 y>l k+y,
$$

and by (4) the number $N(s, l)$ of $A \in \tilde{\mathscr{F}}$ with $\left|A \cap\left(X_{1} \cup \cdots \cup X_{l}\right)\right| \geq l k$ does not exceed

$$
\begin{aligned}
& \left(\begin{array}{c}
\left|X_{1} \cup \cdots \cup X_{l}\right| \\
l k
\end{array}\right) \varphi(n-s y-l k, q) \\
& \leq\left(\begin{array}{c}
l y \\
l k
\end{array}\right) C(q)(n-s y-l k) ![\log \log (n-s y-l k)\rfloor^{4 q \alpha(n-s y-l k, q)} \\
& \quad \times \alpha(n-s y-l k, q)^{s y+l k-n} .
\end{aligned}
$$

Since $n-s y-l k>y$, for large $n$ we have

$$
\begin{aligned}
& \lfloor\log \log (n-s y-l k)\rfloor^{4 q \alpha(n-s y-l k . q)} \alpha(n-s y-l k, q)^{s y+l k-n} \\
& \quad \leq(\log (n-s y-l k))^{1 / 5} \alpha(n-s y-l k, q)^{s y+l k-n}<1
\end{aligned}
$$

Consequently,

$$
N(s, l) \leq\left(\frac{e l y}{l k}\right)^{l k} C(q)(n-s y-l k) ! \leq \frac{C(q)(n-s y) !}{(k / e)^{l k}} .
$$

But for large $n$ we have

$$
\left(\frac{k}{e}\right)^{k} \geq\left(\frac{n}{e \log n}\right)^{\frac{n(\log \log n)^{3}}{\log n}} \geq e^{0.5 n(\log \log n)^{3}}>e^{n(3 q \alpha)^{3}} .
$$

In view of $(6)$, we obtain

$$
k ! \geq(k / e)^{k} \geq\left(2 \xi_{m}\right)^{n}
$$

and for the family $\mathscr{H}^{\prime}:=\left\{A \in \tilde{\mathscr{F}}|| A \cap\left(X_{1} \cup \cdots \cup X_{l}\right) \mid<l k\right\}$ we have $\left|\mathscr{H}^{\prime}\right| \geq$ $|\tilde{\mathscr{F}}|-C(q)(n-s y) !\left(2 \xi_{s}\right)^{s y-n} \geq C(q)(n-s y) !\left(2 \xi_{s}\right)^{s y-n}$. Then by Lemma 3 there exist $\mathscr{H}_{l+1} \subset \mathscr{H}^{\prime}$ and $X_{l+1}$ with $\left|X_{l+1}\right|=y$ such that each $A \in \mathscr{H}_{l+1}$ contains $X_{l+1}$ and $\left|\mathscr{H}_{l+1}\right| \geq C(q)(n-(s+1) y) ! \beta^{(s+1) y-n}$, where

$$
\begin{aligned}
\beta & =\left(2 q \times 2 \xi_{s}\right)^{\frac{n-s y}{n-(s+1) y}}=\left(4 q \times\left((2 \alpha)^{n}(4 q)^{n s-s(s-1) y / 2}\right)^{\frac{1}{n-s y}}\right)^{\frac{n-s y}{n-(s+1) y}} \\
& =\left((2 \alpha)^{n}(4 q)^{n(s+1)-s(s+1) y / 2}\right)^{\frac{1}{n-(s+1) y}}=\xi_{s+1} .
\end{aligned}
$$

By definition of $\mathscr{H}^{\prime}$, 


$$
\left|X_{l+1} \cap\left(X_{1} \cup \cdots \cup X_{l}\right)\right|<l k .
$$

Putting $Z_{l+1}:=Z_{l} \cup\left(X_{l+1} \cap\left(X_{1} \cup \cdots \cup X_{l}\right)\right)$, we have $\left|Z_{l+1}\right| \leq\left|Z_{l}\right|+l k \leq k l(l+$ $1) / 2$ and conditions (7) are fulfilled for $l+1$. Thus we can proceed till $l=r$.

After constructing $\mathscr{H}_{r}, X_{r}$, and $Z_{r}$, we put, for $j=1, \ldots, r$, $X\left(i_{1}, i_{2}, \ldots, i_{s}, j\right):=X_{j}$ and

$$
\mathscr{F}\left(1, i_{1}, \ldots, i_{s}, j\right):=\left\{A \cup X\left(i_{1}\right) \cup X\left(i_{1}, i_{2}\right) \cup \cdots \cup X\left(i_{1}, i_{2}, \ldots, i_{s}, j\right) \mid A \in \mathscr{H}_{i}\right\}
$$

and

$$
Z\left(1, i_{1}, \ldots, i_{s}\right):=Z_{r} \text {. }
$$

By construction, the statements (i)-(vii) of the lemma will be fulfilled for $s+1$

Lemma 5. For all $s=0,1, \ldots, m+1$ and for all $i_{1}, \ldots, i_{s} \in\{1, \ldots, r\}$, there are sets $X\left(i_{1}, \ldots, i_{s}\right)$ and $Z\left(1, i_{1}, \ldots, i_{s-1}\right)$ and for all $i_{1}, \ldots, i_{m+1} \in\{1, \ldots, r\}$, there are sets $A\left(i_{1}, \ldots, i_{m+1}\right) \in \mathscr{F}$ such that

(i) the sets $X\left(i_{1}\right), X\left(i_{1}, i_{2}\right), \ldots, X\left(i_{1}, i_{2}, \ldots, i_{m+1}\right)$ are pairwise disjoint;

(ii) $\left|X\left(i_{1}, i_{2}, \ldots, i_{s}\right)\right|=y$ if $1 \leq s \leq m$,

(iii) $\left|X\left(i_{1}, i_{2}, \ldots, i_{m+1}\right)\right|=n-m y$,

(iv) $A\left(i_{1}, \ldots, i_{m+1}\right)=X\left(i_{1}\right) \cup X\left(i_{1}, i_{2}\right) \cup \cdots \cup X\left(i_{1}, i_{2}, \ldots, i_{m+1}\right)$, for all $s=$ $1, \ldots, m$ and for all $i_{1}, \ldots, i_{s}, i_{s}^{\prime} \in\{1, \ldots, r\}$,

(v) $X\left(i_{1}, i_{2}, \ldots, i_{s-1}, i_{s}\right) \cap X\left(i_{1}, i_{2}, \ldots, i_{s-1}, i_{s}^{\prime}\right) \subset Z\left(1, i_{1}, i_{2}, \ldots, i_{s-1}\right)$,

(vi) $\left|Z\left(1, i_{1}, i_{2}, \ldots, i_{s-1}\right)\right| \leq k(r+1) / 2$.

Proof. For $s=0,1, \ldots, m$ and for any $i_{1}, \ldots, i_{s} \in\{1, \ldots, r\}$, consider $\mathscr{F}\left(1, i_{1}, \ldots, i_{s}\right), X\left(i_{1}, \ldots, i_{s}\right)$, and $Z\left(1, i_{1}, \ldots, i_{s-1}\right)$ from Lemma 4.

Now, for an arbitrary $(m+1)$-tuple $\left(1, i_{1}, \ldots, i_{m}\right)$, consider

$$
\begin{aligned}
\mathscr{H}= & \mathscr{H}\left(1, i_{1}, \ldots, i_{m}\right) \\
& :=\left\{A \backslash\left(X\left(i_{1}\right) \cup X\left(i_{1}, i_{2}\right) \cup \cdots \cup X\left(i_{1}, i_{2}, \ldots, i_{m}\right)\right) \mid A \in \mathscr{F}\left(1, i_{1}, \ldots, i_{m}\right)\right\} .
\end{aligned}
$$

By construction, $\mathscr{H}$ is a $(q, n-m y, 1, q)$-family and, by Lemma $4,|\mathscr{H}| \geq C(n-$ $m y) ! \xi_{m}^{m y-n}$.

Recall that $n-m y \geq n /(3 \alpha)$. By (8), for large $n,|\mathscr{H}|>C(q)(n-m y) ! / k !$ and by Lemma 2 [note that $0<r<k<(n-m y) / 2$ ], there exist $A_{1}, \ldots, A_{r} \in \mathscr{H}$ such that

$$
\left|A_{1} \cup \cdots \cup A_{r}\right|>r(n-m y)-k r^{2} / 2
$$

Let

$$
Z\left(1, i_{1}, \ldots, i_{m}\right):=\bigcup_{1 \leq j<h \leq r} A_{j} \cap A_{h},
$$

and for $j=1, \ldots, r, X\left(i_{1}, i_{2}, \ldots, i_{m}, j\right):=A_{j}$, and

$$
A\left(i_{1}, \ldots, i_{m}, j\right)=X\left(i_{1}\right) \cup X\left(i_{1}, i_{2}\right) \cup \cdots \cup X\left(i_{1}, i_{2}, \ldots, i_{m}\right) \cup A_{j} .
$$

In view of (9), $\left|Z\left(1, i_{1}, \ldots, i_{m}\right)\right| \leq k r^{2} / 2$. Now, by Lemma 4 and the construction, all the statements of the lemma are fulfilled. 
Lemma 6. If $A \in \mathscr{F}$ and $A \cap A\left(i_{1}, \ldots, i_{m+1}\right) \neq \emptyset$ for all $i_{1}, \ldots, i_{m+1} \in$ $\{1, \ldots, r\}$, then there exist $s, 0 \leq s \leq m$ and $i_{1}, \ldots, i_{s} \in\{1, \ldots, r\}$ such that

$$
A \cap X\left(i_{1}, i_{2}, \ldots, i_{s}, j\right) \neq \emptyset \quad \forall j \in\{1, \ldots, r\} .
$$

Proof. Assume that for some $B \in \mathscr{F}$ for each $s, 0 \leq s \leq m$ and each $i_{1}, \ldots, i_{s} \in$ $\{1, \ldots, r\}$, there exists $j^{*}\left(1, \ldots, i_{s}\right)$ such that

$$
B \cap X\left(i_{1}, i_{2}, \ldots, i_{s}, j^{*}\left(1, \ldots, i_{s}\right)\right)=\emptyset .
$$

Let further $q_{0}=1$ and for $s=1, \ldots, m+1, q_{s}=j^{*}\left(q_{0}, \ldots, q_{s-1}\right)$. Then $B$ has empty intersection with every member of the sequence $X\left(q_{1}\right), X\left(q_{1}, q_{2}\right), \ldots, X\left(q_{1}, q_{2}, \ldots, q_{m+1}\right)$. But this means that $B$ is disjoint from $A\left(q_{1}, q_{2}, \ldots, q_{m+1}\right)$, and we are done

Completion of the Proof of the Theorem. Consider

$$
Z:=\bigcup_{s=1}^{m+1} \bigcup_{\left(1, i_{1}, \ldots, i_{s-1}\right)} Z\left(1, i_{1}, \ldots, i_{s-1}\right) .
$$

Clearly, $|Z| \leq\left(1+r+r^{2}+\cdots+r^{m}\right) k r(r+1) / 2 \leq k r^{m+2}=k r^{3 \alpha+1}$.

Denote by $\mathscr{B}\left(i_{1}, \ldots, i_{m+1}\right)$ the collection $\left\{A \in \mathscr{F} \mid A \cap 4\left(i_{1}, \ldots, i_{m+1}\right)=\emptyset\right\}$.

Let $\mathscr{E}=\{A \in \mathscr{F} \mid A \cap Z \neq \emptyset\}$ and

$$
\mathscr{H}\left(1, i_{1}, \ldots, i_{s}\right)=\left\{A \in \mathscr{F} \backslash \mathscr{E} \mid A \cap X\left(i_{1}, i_{2}, \ldots, i_{s}, j\right) \neq \emptyset \forall j \in\{1, \ldots, r\}\right\} .
$$

By Lemma 5 , for each $s, 0 \leq s \leq m$ and $i_{1}, \ldots, i_{s} \in\{1, \ldots, r\}$, the sets $X\left(i_{1}, i_{2}, \ldots, i_{s}, j\right) \backslash Z$ for distinct $j$ are pairwise disjoint. Hence by Lemma 6 , we can write $\mathscr{F}$ in the form

$$
\mathscr{F}=\bigcup_{\left(i_{1}, \ldots, i_{m+1}\right)} \mathscr{B}\left(i_{1}, \ldots, i_{m+1}\right) \cup \mathscr{E} \cup\left(\bigcup_{s=0}^{m} \bigcup_{\left(1, i_{1}, \ldots, i_{s}\right)} \mathscr{H}\left(1, i_{1}, \ldots, i_{s}\right)\right) .
$$

By (3),

$$
\left|\bigcup_{\left(i_{1}, \ldots, i_{m+1}\right)} \mathscr{B}\left(i_{1}, \ldots, i_{m+1}\right)\right| \leq r^{m+1} C(q) n ! r^{4 \alpha(n, q)(p-1)} \alpha(n, q)^{-n}
$$

Then,

$$
|\mathscr{E}| \leq|Z| \varphi(n-1, q) \leq k r^{3 \alpha+1} C(q)(n-1) ! r^{4 q \alpha(n-1, q)} \alpha(n-1, q)^{1-n} .
$$

Note that each $A \in \mathscr{H}\left(1, i_{1}, \ldots, i_{s}\right)$ must intersect each of $r$ pairwise disjoint sets $X\left(i_{1}, i_{2}, \ldots, i_{s}, 1\right) \backslash Z, X\left(i_{1}, i_{2}, \ldots, i_{s}, 2\right) \backslash Z, \ldots, X\left(i_{1}, i_{2}, \ldots, i_{s}, r\right) \backslash Z$. The cardinalities of these sets for $s<m$ are at most $y$ and for $s=m$ are less than $2 y$. Consequently, by (4), $\left|\mathscr{H}\left(1, i_{1}, \ldots, i_{s}\right)\right| \leq(2 y)^{r} \varphi(n-r, q) \leq\left(\frac{2 n}{3 \alpha}\right)^{r} C(q)(n-r) ! r^{4 q \alpha(n-r, q)} \alpha(n-r, q)^{r-n}$.

Observe that, for $n>20$ and $1 \leq i \leq 0.5 \log n$, 


$$
\begin{aligned}
& \frac{\alpha(n-i, q)}{\alpha(n, q)} \geq \frac{\log \log (n-i)}{\log \log n} \geq \frac{\log (\log n-i /(n-i))}{\log \log n} \\
& \quad \geq \frac{\log ((1-1 /(n+1)) \log n)}{\log \log n} \geq \frac{\log \log n-1 / n}{\log \log n} \geq 1-\frac{1}{n} .
\end{aligned}
$$

Therefore,

$$
\begin{aligned}
|\mathscr{F}| \leq & C(q) n ! r^{(4 p-1) \alpha(n, q)} \alpha(n, q)^{-n}+k r^{3 \alpha+1} C(q)(n-1) ! r^{4 q \alpha(n-1, q)} \\
& \times((n-1) \alpha(n, q) / n)^{1-n} \\
& +\frac{r^{m+1}-1}{r-1}\left(\frac{2}{2.8 \alpha(n, q)}\right)^{r} C(q) n ! r^{4 q \alpha(n-r, q)}\left(\frac{n-1}{n} \alpha(n, q)\right)^{r-n} \\
< & C(q) n ! \alpha(n, q)^{-n} r^{4 p \alpha(n, q)}\left(r^{-\alpha(n, q)}+\frac{3(\log \log n)^{4 q \alpha(n, q)+3} \alpha(n, q)}{\log n}\right. \\
& \left.+\frac{3(\log \log n)^{4 q \alpha(n, q)}}{1.4^{(\log \log n\rfloor}}\right) .
\end{aligned}
$$

But for large $n$ the expression in big parentheses does not exceed

$$
(\log n)^{-1 / 10 q}+3(\log \log n)^{4}(\log n)^{-4 / 5}+4 / 2(\log n)^{0.2-\log 1.4}<1 .
$$

Thus, the theorem is proved.

\section{ACKNOWLEDGMENT}

The author is very indebted to $M$. Axenovich and D. G. Fon-Der-Flaass for many creative conversations.

\section{REFERENCES}

[1] H. L. Abbott, D. Hanson, and N. Sauer, Intersection theorems for systems of sets, $J$. Combinat. Theor., A12, 381-389 (1972).

[2] P. Erdôs, Problems and results on set systems and hypergraphs, in Bolyai Society Mathematical Studies, 3, Extremal Problems for Finite Sets, Visegrad, Hungary, 1991, pp. 217-227.

[3] P. Erdös and R. Rado, Intersection theorems for systems of sets, J. London Math. Soc. 35, 85-90 (1960).

[4] A. V. Kostochka, A bound of the cardinality of families not containing $\Delta$-systems, in The Mathematics of Paul Erdós, R. L. Graham and J. Nešetrill, Eds., to appear.

[5] J. Spencer, Intersection theorems for systems of sets, Canad. Math. Bull., 20, 249-254 (1977). 